\title{
Progress in the Calculation of Parton Distributions in QCD
}

\author{
A. W. Thomas ${ }^{\mathrm{a}}$, W. Detmold ${ }^{a}$ and W. Melnitchouk ${ }^{a b}$ \\ ${ }^{a}$ Special Research Centre for the Subatomic Structure of Matter, and Department of \\ Physics and Mathematical Physics, Adelaide University, 5005, Australia \\ ${ }^{\mathrm{b}}$ Jefferson Lab, 12000 Jefferson Avenue, Newport News, VA 23606 USA
}

Until recently state of the art studies of the moments of parton distributions within lattice QCD have led to discrepancies with experimental data at the level of $50 \%$. Such a large disagreement in a fundamental hadronic observable casts doubt on the attempt to calculate all hadron properties on the lattice. We summarise a recent discovery, concerning the need to correctly incorporate chiral symmetry into the extrapolation to physical quark masses, which removes this discrepancy. In so doing it also opens the exciting possibility that using similar techniques for other observables one may be able to make reliable comparisons with experimental data using the next generation of supercomputers, available within the next two or three years.

\section{Introduction}

Recent discoveries concerning the nucleon's parton distribution functions (PDFs) - such as the proton "spin crisis" [1], the SU(2) flavor symmetry violation of the proton sea [2] and the nuclear EMC effect [3] - have had an important impact on our understanding of the structure of hadrons. Future experiments will explore further aspects of the nonperturbative origin of parton distributions and test theoretical predictions, thereby deepening that understanding. While they are not accessible in perturbation theory, PDFs have been investigated in numerous QCD-motivated quark models, and some important model independent results [4] exist. However, one would ultimately like to explore these phenomena within QCD itself.

Currently, lattice field theory is the only quantitative tool available for the calculation of hadronic properties which is based on QCD from first principles. It is therefore somewhat shocking that naive extrapolation of the moments of PDFs, calculated within existing lattice simulations, typically lie some $50 \%$ above the experimental data at the physical quark mass. Here we present the resolution of this discrepancy based on an analysis [5] of lattice QCD data on the first three nontrivial moments of the nonsinglet parton distribution $u-d$, which includes a consistent chiral extrapolation of the moments to the physical region. 


\section{Lattice calculations of structure function moments}

Let us define the moments of the PDFs as:

$\left\langle x^{n}\right\rangle_{q}=\int_{0}^{1} d x x^{n}\left(q\left(x, Q^{2}\right)+(-1)^{n+1} \bar{q}\left(x, Q^{2}\right)\right)$,

where the quark distribution $q\left(x, Q^{2}\right)$ is a function of the Björken scaling variable $x$ (at momentum scale $Q^{2}$ ). Then the operator product expansion relates these moments to the forward nucleon matrix elements of certain local twist- 2 operators which can be accessed in lattice simulations [5].

Early calculations of moments of structure functions within lattice QCD were performed by Martinelli and Sachrajda[6]. However, the more recent data used in this analysis are taken from the QCDSF [7] and MIT [8] groups and shown in Fig. 1 for the $n=1,2$ and 3 moments of the $u-d$ difference at NLO in the $\overline{\mathrm{MS}}$ scheme. These calculations have been performed for both full and quenched QCD using a variety of quark actions and for quark masses, $m_{q}$, ranging from 50 to $190 \mathrm{MeV}$.

To compare the lattice results with the experimentally measured moments, one must extrapolate the data from the lowest quark mass used $(\sim 50 \mathrm{MeV})$ to the physical value $(\sim 5-6 \mathrm{MeV})$. Naively this is done by assuming that the moments depend linearly on the quark mass. However, as shown in Fig. 1 (long dashed lines), a linear extrapolation of the world lattice data for the $u-d$ moments typically overestimates the experimental values by $50 \%$. This suggests that important physics is still being omitted from the lattice calculations and their extrapolations.

\subsection{Nonanalytic behaviour}

One knows on very general grounds that a linear extrapolation in $m_{q} \sim m_{\pi}^{2}$ must fail as it omits crucial nonanalytic structure associated with chiral symmetry breaking. Even at the lowest quark mass accessed on the lattice, the pion mass is over $300 \mathrm{MeV}$. Earlier studies of chiral extrapolations of lattice data for hadron masses [10], magnetic moments [11] and charge radii [12] have shown that for quark masses above $50-60 \mathrm{MeV}$, hadron properties behave very much as one would expect in a constituent quark model, with relatively smooth behaviour as a function of the quark mass. However, for $m_{q}<50 \mathrm{MeV}$ one typically finds rapid, nonlinear variation arising from the nonanalytic behaviour of Goldstone boson loops [13].

In general, contributions to the physical properties of hadrons from intermediate states involving the surrounding meson cloud give rise to unique terms which are nonanalytic in the quark mass. These stem from the infra-red behaviour of the chiral loops and are model independent. The leading nonanalytic (LNA) term for the $u$ and $d$ distributions in the physical nucleon arises from the single pion loop dressing of the bare nucleon and has been shown $[4,15]$ to behave as:

$\left\langle x^{n}\right\rangle_{q}^{\mathrm{LNA}} \sim m_{\pi}^{2} \log m_{\pi}$.

\subsection{Improved chiral extrapolation}

Experience with the chiral behaviour of masses and magnetic moments shows that the LNA terms alone are not sufficient to describe lattice data for $m_{\pi}>200 \mathrm{MeV}[10,11]$. 


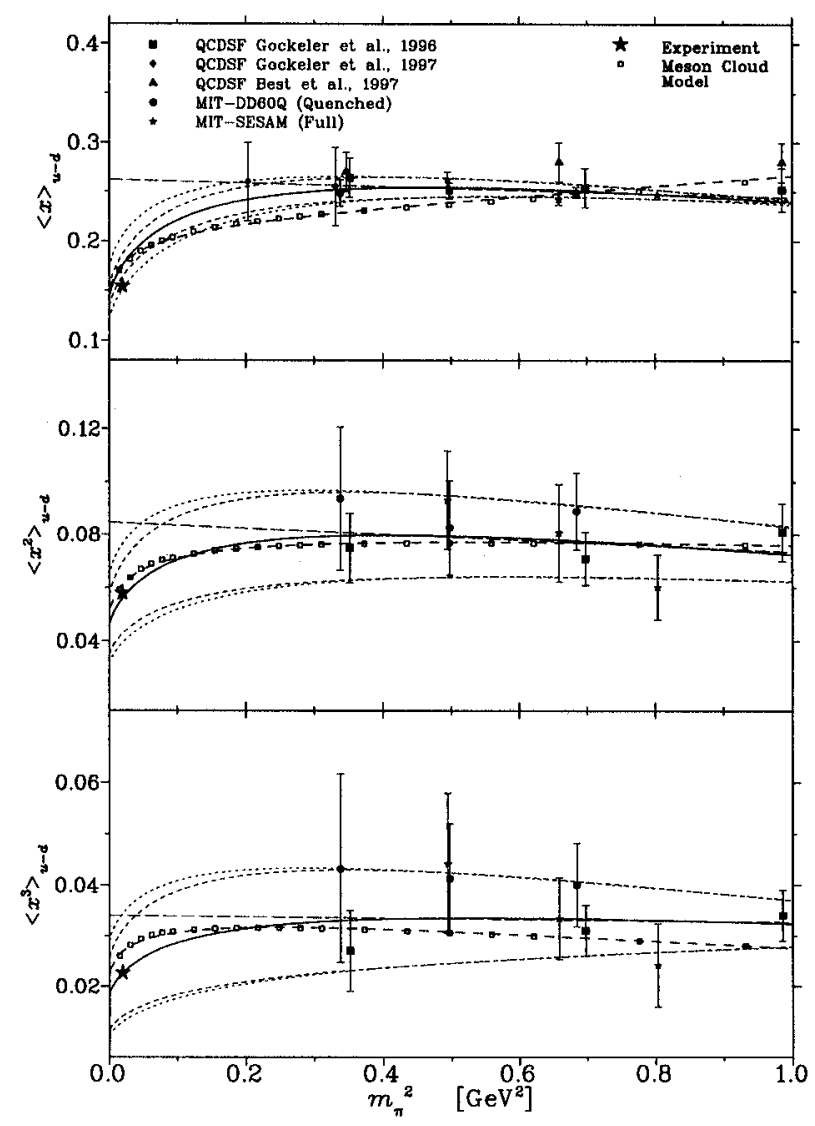

Figure 1. Moments of the $u-d$ quark distribution from various lattice simulations. The straight (long-dashed) lines are linear fits to this data, while the curves have the correct LNA behaviour in the chiral limit - see the text for details. The small squares are the results of the meson cloud model and the dashed curve through them best fits using Eq. (3). The star represents the phenomenological values taken from NLO fits [9] in the $\overline{\mathrm{MS}}$ scheme. 
Thus, in order to fit the lattice data at larger $m_{\pi}$ while preserving the correct chiral behaviour of moments as $m_{\pi} \rightarrow 0$, a low order, analytic expansion in $m_{\pi}^{2}$ is also included in our extrapolation and the moments of $u-d$ are fitted with the form:

$\left\langle x^{n}\right\rangle_{u-d}=a_{n}+b_{n} m_{\pi}^{2}+a_{n} c_{\mathrm{LNA}} m_{\pi}^{2} \ln \left(\frac{m_{\pi}^{2}}{m_{\pi}^{2}+\mu^{2}}\right)$,

where the coefficient $c_{\mathrm{LNA}}=-\left(3 g_{A}^{2}+1\right) /\left(4 \pi f_{\pi}\right)^{2}$ [15]. The parameters $a_{n}, b_{n}$ and $\mu$ are a priori undetermined. The mass $\mu$ determines the scale above which pion loops no longer yield rapid variation and corresponds to the upper limit of the momentum integration if one applies a sharp cut-off in the pion loop integral. Multi-meson loops and other contributions cannot give rise to the LNA behaviour in Eq. (2) and thus near the chiral limit Eq. (3) is the most general form for moments of the PDFs at $\mathcal{O}\left(m_{\pi}^{2}\right)$ which is consistent with chiral symmetry. While the current lattice data are at values of $m_{\pi}$ too high to display any deviation from constituent quark behaviour, it is not obvious why a lowest order form should be able to fit data up to $m_{\pi} \sim 1 \mathrm{GeV}$. Studies of the $N$ and $\Delta$ masses [10] and the nucleon magnetic moments [11] indicate that the cloudy bag model [16] (CBM) can describe the corresponding lattice data over a wide range of quark mass. For the structure functions we used a similar approach with the nucleon modelled as a bag or a bag plus a pion. The details [17] of structure function calculations in this model are well known, and since the model is not our main focus, we simply present the results for the first three moments in Fig. 1. The small squares are the results of the model calculation while the dashed curves through them are $\chi^{2}$ fits to these using the form given in Eq. (3). Clearly, this form provides an excellent fit to the data generated by the model, which are also in semi-quantitative agreement with the calculated lattice moments. These results give us confidence that a fit to the lattice data based on Eq. (3) should be reasonable.

Having motivated the functional form of the extrapolation formula, we now apply Eq. (3) to the lattice data. Unfortunately, data are not yet available at quark masses low enough to allow a reliable determination of the mass parameter $\mu$. Consequently, for the central curve in each panel of Fig. 1 the value that is most consistent with all experimental moments was chosen, $\mu=550 \mathrm{MeV}$. With $\mu$ thus fixed, the results of the best $\chi^{2}$ fit (for parameters $a_{n}$ and $b_{n}$ ) to the lattice data for each moment are given by the central solid lines. To estimate the error in the extrapolated value (for a fixed $\mu$ ), we also fit to the extrema of the error bars on the data as is shown in Fig. 1 by the inner envelopes around these curves.

Experience with other hadronic properties, such as magnetic moments and masses, suggests that the switch (as a function of current quark mass) from smooth and constituent quark-like behaviour (slowly varying with respect to the current quark mass) to rapidly variation (dominated by Goldstone boson loops) happens for $m_{\pi} \sim 500-600 \mathrm{MeV}$. This is very close to the preferred value of $\mu$ found here and the similarity of these scales for the various observables simply reflects the common scale at which the Compton wavelength of the pion becomes comparable to the size of the bare hadron. This value of $\mu$ is also similar to the value required by the $\chi^{2}$ fits to the meson cloud model. Nevertheless, we stress that $\mu$ is not yet determined by the lattice data and it is indeed possible to consistently fit both the lattice data and the experimental values with $\mu$ ranging from $400 \mathrm{MeV}$ to 
$700 \mathrm{MeV}$. This dependence on $\mu$ is illustrated in Fig. 1 by the difference between the inner and outer envelopes on the fits. The former are the best fits to the lower (upper) limits of the error bars, while the latter use $\mu=450$ (650) $\mathrm{MeV}$ instead of the central value of $\mu=550 \mathrm{MeV}$. Data at smaller quark masses are therefore crucial to constrain this parameter and perform an accurate extrapolation.

\section{Study of Parton Distribution Functions versus Quark Mass}

Since the main physical interest is in the PDFs, rather than the moments, we wish to see to what extent the lowest moments can be used to reconstruct the underlying distributions. In order to compare with standard parameterisations [18], the form

$x q(x)=\alpha x^{b}(1-x)^{c}(1+\epsilon \sqrt{x}+\gamma x)$,

is assumed for the distribution. Correspondingly the $n^{\text {th }}$ moment will be given by

$\left\langle x^{n}\right\rangle_{u-d}=\alpha[B(1+c, b+n)+\epsilon B(1+c, 0.5+b+n)+\gamma B(1+c, 1+b+n)]$,

where $B(x, y)$ is the $\beta$-function. Leaving the parameters $\epsilon$ and $\gamma$ fixed to their MRS values (see Ref. 14 for further details), the extrapolated lattice moments are used to fit the parameters $b$ and $c$ ( $\alpha$ is fixed by requiring $\left\langle x^{0}\right\rangle_{u-d}=1$ ). This procedure is performed at the physical quark mass using both the naive, linear extrapolation and the improved chiral extrapolation, with vastly differing results as shown in Fig. 2. In particular, whereas the correct chiral extrapolation yields close agreement with MRS, the linear extrapolation leads to a distribution peaking near $x=1 / 3$ - as expected in a constituent quark model.

\section{Conclusion}

We have investigated the moments of the $u-d$ distribution as a function of quark mass, paying particular attention to the behaviour in the chiral limit. As found earlier for hadron masses, magnetic moments and charge radii, at large quark mass the moments of structure functions exhibit only slowly varying, linear dependence on $m_{q}$. However, when the mass decreases towards the chiral limit, this dependence becomes increasingly nonlinear as the effects of pion loops become dominant. The transition happens when the pion Compton wavelength becomes larger than the pion source.

A low order formula for the mass dependence of the moments has been proposed, which embodies the LNA behaviour expected from the chiral properties of QCD, and this has been used to extrapolate the available lattice data to the physical region. The applicability of a low order expansion for the lattice data is supported by phenomenological chiral quark model studies. Compared with linear extrapolations, which badly overestimate the experimental values, the improved extrapolation presented here shows that, within the current errors, there is no evidence of a discrepancy between the lattice data and experiment once the correct dependence on quark mass near the chiral limit is incorporated. However, in order to properly constrain the extrapolation purely from data, statistically compelling lattice calculations are needed at quark masses $\sim 20 \mathrm{MeV}$. This is an exciting result in that it should be possible to carry out full QCD simulations in this mass 


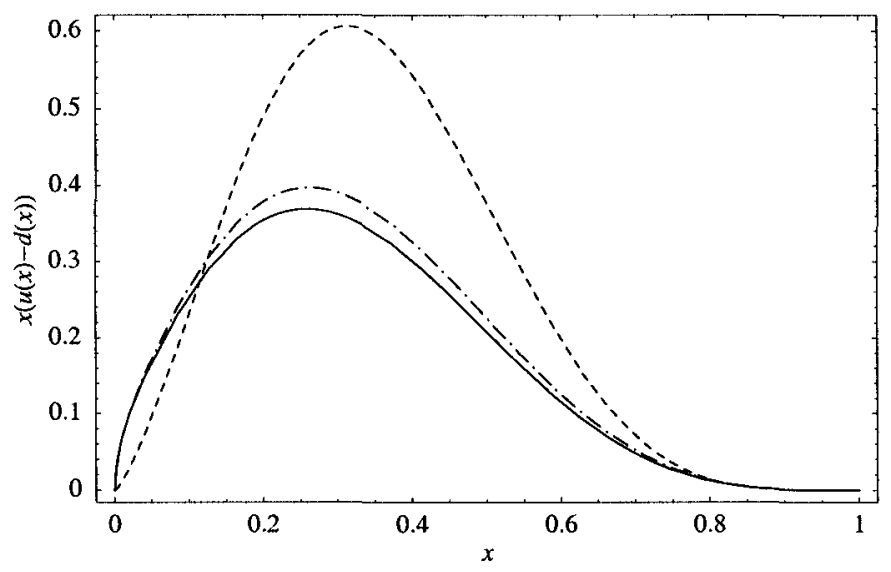

Figure 2. The PDF $x(u(x)-d(x))$ is shown at the physical quark mass as extracted using a linear extrapolation of the lattice data (dashed curve) and using the improved chiral extrapolation (solid curve). For comparison we also show the MRS [18] parameterisation (dashed-dotted curve).

region on the next generation of supercomputers, available in the next two or three years, rather than waiting a decade for the machines capable of 100 's Tera-flops needed to make calculations with the physical quark masses.

Finally, we have also shown that using just the lowest four moments of $u-d$ one can reconstruct the underlying PDF with reasonable accuracy. The study of the shape of this distribution as a function of quark mass reveals that, provided the correct chiral extrapolation is employed, the resulting distribution is very close to that determined from deep inelastic scattering data. On the other hand, the PDF reconstructed from a naive linear extrapolation of the moments is very similar to that expected in a constituent quark model.

\section{Acknowledgments}

We would like to acknowledge the important contributions of our collaborators in the work concerning improved chiral extrapolation, D. B. Renner and J. W. Negele. We are also grateful to D. Leinweber for many useful discussions concerning the chiral properties of lattice QCD. This work was supported by the Australian Research Council and Adelaide University.

\section{REFERENCES}

1. J. Ashman et al., Phys. Lett. B 206, 364 (1988). 
2. P. Amaudruz et al., Phys. Rev. Lett. 66, 2712 (1991); E.A. Hawker et al., Phys. Rev. Lett. 80, 3715 (1998).

3. J.J. Aubert et al., Phys. Lett. B 123275 (1983).

4. A.W. Thomas, W. Melnitchouk and F.M. Steffens, Phys. Rev. Lett. 85, 2892 (2000).

5. W. Detmold et al., hep-lat/0103006, submitted to Phys. Rev. Lett.

6. G. Martinelli and C.T. Sachrajda, Phys. Lett. B 196, 184 (1987); Nucl. Phys. B306, 865 (1988).

7. M. Göckeler et al., Phys. Rev. D 53, 2317 (1996); M. Göckeler et al., Nucl. Phys. Proc. Suppl. 53, 81 (1997); C. Best et al., hep-ph/9706502.

8. D. Dolgov et al., hep-lat/0011010, and to be published; D. Dolgov, Ph.D. thesis, MIT, Sep. 2000.

9. H.L. Lai et al., Eur. Phys. J. C 12, 375 (2000); A.D. Martin et al., Eur. Phys. J. C 14, 133 (2000); M. Gluck, E. Reya and A. Vogt, Eur. Phys. J. C 5, 461 (1998).

10. D.B. Leinweber, A.W. Thomas, K. Tsushima and S.V. Wright, Phys. Rev. D 61, $074502(2000)$.

11. D.B. Leinweber et al., Phys. Rev, D 60, 034014 (1999); D.B. Leinweber and A.W. Thomas, ibid. D 62, 074505 (2000); E.J. Hackett-Jones et al., Phys. Lett. B 489, $143(2000)$.

12. M.A.B. Bég and A. Zepeda, Phys. Rev. D 6, 2912 (1972); J. Gasser, M.E. Sainio and A. Svarc, Nucl. Phys. B 307, 779 (1988); D.B. Leinweber and T.D. Cohen, Phys. Rev. D 47, 2147 (1993); E.J. Hackett-Jones, D.B. Leinweber and A.W. Thomas, Phys. Lett. B 494, 89 (2000).

13. S. Weinberg, Physica (Amsterdam) 96 A, 327 (1979); J. Gasser and H. Leutwyler, Ann. Phys. 158, 142 (1984).

14. W. Detmold et al., in preparation.

15. D. Arndt and M.J. Savage, nucl-th/0105045; J.-W. Chen and X. Ji, hep-ph/0105197.

16. S. Théberge, G.A. Miller and A.W. Thomas, Phys. Rev. D 22, 2838 (1980); A.W. Thomas, Adv. Nucl. Phys. 13, 1 (1984).

17. A.W. Schreiber, A.I. Signal and A.W. Thomas, Phys. Rev. D 44, 2653 (1991);

J. Speth and A. W. Thomas, Adv. Nucl. Phys. 24, 83 (1997);

C. Boros and A.W. Thomas, Phys. Rev. D 60, 074017 (1999).

18. A.D. Martin, et al., Phys. Rev. D 50, 6734 (1994). 